\title{
Tendencias curriculares en Astronomía Cultural
}

Javier Mejuto

\section{Resumen}

El presente trabajo realiza un recorrido por la situación académica de la disciplina desde sus primeros pasos hasta el presente proyectando el futuro de la misma en los próximos años. El objetivo es su presencia fuerte y definitiva en los centros de educación superior. Se realiza un análisis global de los diferentes planes de estudios -tanto en educación formal como no formal- en Astronomía Cultural y disciplinas afines para conocer las tendencias y materias que están conformando en la actualidad el cuerpo de conocimientos y competencias de los egresados de estos estudios. Debido a la baja inclusión en el ambiente académico de la disciplina en todo el mundo se propone la creación de unos estudios de licenciatura en Astronomía Cultural como el siguiente paso necesario para el desarrollo de la disciplina.

Palabras claves: Astronomía Cultural, Arqueoastronomía, Desarrollo curricular.

\section{Abstract}

This paper provides an overview of the academic status of the discipline, from its beginning to the present, to project the future of the discipline in the coming years which should be marked by a strong and permanent presence in higher education. A comprehensive analysis between Cultural Astronomy different studies - in formal and non-formal education- is conducted in order to know the trends and subjects that are shaping today's body of knowledge and skills of the graduates of these studies. Due to the discipline's low inclusion in the academic environment worldwide creating a Degree in Cultural Astronomy as the next necessary for the development of the discipline step is proposed.

Keywords: Cultural Astronomy, (javier.mejuto@unah.edu.hn) Archaeoastronomy, Curriculum Development.

Javier Mejuto, Departamento de Arqueoastronomía y Astronomía Cultural. Facultad de Ciencias Espaciales Universidad Nacional Autónoma de Honduras 


\section{Introducción}

Se considera tradicionalmente que la Astronomía Cultural -usando la definición disciplinar de Iwaniszewski (Iwaniszewski, 1991), aunque el término ha ido cambiando desde su denominación como astroarqueología (Iwaniszewski, 1994)comienza con el trabajo de Sir Norman Lockyer y, en particular, con su libro The Dawn of Astronomy en 1894. A pesar de que ya han pasado más de 100 años, se sigue asumiendo que la Astronomía Cultural es una ciencia emergente y muy joven en comparación con otras ciencias. Aunque cabría pensar que en los 120 años que han pasado desde la publicación del libro de Lockyer el desarrollo de la disciplina ha sido lineal, basta consultar algunas recensiones históricas (Baity, 1973) o la evolución del mismo término arqueoastronomía para darse cuenta de lo contrario, siendo en ocasiones incluso aparcada por décadas. Sin embargo, este trabajo no busca ocuparse tanto de la historia disciplinar como de su presente y muy especialmente de su futuro dada su emergencia y juventud.

Existen varios momentos clave que han ido conformando el campo. En particular se han marcado una serie de tendencias que se desarrollaron con las primeras investigaciones en el área y que han permanecido vigentes hasta la actualidad. El primer punto de interés es la preponderancia de los estudios sobre megalitismo en Europa, plasmada en trabajos icónicos como Stonehenge and other british monuments astronomically considered del mismo Lockyer, que han relegado a otros contextos culturales a un plano muy secundario en la Astronomía Cultural. Afortunadamente, la expansión de las investigaciones a otros países ha diversificado y aumentado -aunque no de manera suficiente, especialmente en número- la cantidad de trabajos sobre contextos arqueológicos: Neolítico, Calcolítico, Bronce, Edad del Hierro, etcétera. A la par del interés por el megalistismo aparece otra tendencia, la geofocalización de los trabajos dentro del área geográfica cercana al investigador que los realiza. Esta tendencia que puede parecer lógica, no se da con igual intensidad en disciplinas afines como la astronomía, arqueología, antropología o etnología.

Inducido por el trabajo de Thom en los años 60 del siglo XX también se ha ido conformando en la Astronomía Cultural europea una preponderancia de la metodología sobre los aspectos culturales de los pueblos que se estudian. Este ha sido un punto de desencuentro en la disciplina entre el viejo y el nuevo mundo, éste último mucho más preocupado por los aspectos culturales de los estudios, dando lugar a la famosa distinción entre la Arqueoastronomía marrón y verde. En América la disciplina goza de una gran salud resultado de la repercusión que han tenido los 
trabajos sobre las principales culturas americanas entre los investigadores arqueólogos con los que a menudo se han realizado trabajos interdisciplinares de gran relevancia e interés, sin embargo debemos tener cuidado de no caer en los vicios ya expuestos con el megalitismo europeo y que otras culturas caigan en el olvido científico.

Afortunadamente las investigaciones tanto en América como en Europa están convergiendo hacia trabajos metodológicamente consistentes aunando un fuerte componente cultural. En relación con la metodología propiamente dicha, no ha ayudado el hecho de que los trabajos hayan venido de la mano de profesionales de ramas tan dispares como geología, astronomía, topografía, ingeniería civil, arqueología,... Todas estas disciplinas han venido acompañadas de su propia metodología de trabajo y han provocado cierto grado de caos metodológico que también ha venido acompañado por su equivalente teórico. En la actualidad -desde un punto de vista académico- la situación de la disciplina es halagüeña pero insuficiente. Existen una cantidad creciente de investigadores que trabajan en el campo desde las universidades y centros de investigación pero sin tener una posición real como campo del conocimiento en dichas universidades. En muchas ocasiones la actividad investigadora en Astronomía Cultural se realiza de forma marginal al campo principal en el que trabajan los investigadores. Esto se refleja en el pequeño impacto que tiene en la docencia universitaria la Astronomía Cultural y de la luego hablaremos.

Los profesionales de la Astronomía Cultural también se encuentran con la necesidad de un medio eficaz y reconocido dónde publicar sus trabajos especializados, efectivamente existen algunas publicaciones pero son de publicación bastante aperiódica en su mayoría. Igualmente valiosa es la creciente creación de redes profesionales que avalen a sus miembros y permitan a los mismos compartir sus experiencias posibilitando el desarrollo de trabajos cada vez más estandarizados y de mayor calidad.

Los problemas esbozados reflejan una disciplina joven, que no acaba de encontrar su lugar entre las ciencias sociales y naturales, entre disciplinas ya consolidadas desde hace tiempo. Uno de los pasos necesarios para consolidarse es encontrar un sitio en el mundo académico universitario. Por lo tanto, es necesario realizar una reflexión que apunte a un desarrollo curricular, científico y ocupacional en Astronomía Cultural. Podría argumentarse que es suficiente con una serie de cursos de posgrado, especialización o tecnificación que completen la formación inicial. Esta posibilidad continuaría creando profesionales con formación heterogénea -en exceso y con vacíos conceptuales en numerosas ocasiones- que seguiría 
impidiendo un desarrollo apropiado disciplinar a través de la discusión epistemológica bajo los mismos parámetros científicos que conlleve a una definición unívoca los objetivos y finalidades de esta rama científica. Es por ello que en este trabajo se propone la necesidad de desarrollar una línea curricular en grado de licenciatura bajo la seguridad de que la misma permitirá homogeneizar a los futuros profesionales.

Si conseguimos que todos estos problemas, ya endémicos de la disciplina, se solucionen a través de una formación específica adecuada sin duda podremos asistir a la consolidación del campo como uno de los de mayor proyección actualmente.

\section{Metodología}

Se ha pretendido recopilar, comparar y analizar los diferentes grados que existen en la actualidad a nivel internacional en Astronomía Cultural. Se han evaluado una serie de parámetros que se han considerado relevantes para completar un análisis final de los mismos.

En particular, se han analizado comparativamente si existen conocimientos y/o estudios requeridos para ingresar a los estudios y en su caso de cuales se trata. Para ello se han analizado las asignaturas, grados o conocimientos previos que son requeridos como base para cursar los programas en estudio.

Se analizaron los perfiles de los estudiantes, aptitudes, competencias y/o habilidades que se requieren para cursar los programas en estudio. Estos mismos programas de estudios se analizaron comparativamente junto con los currículos asociados.

Se ha puesto especial hincapié en las disciplinas y ramas científicas asociadas a los estudios. Se han analizado la existencia de ciencias afines y diferentes áreas del conocimiento, identificando cuáles son. Asimismo se han ubicado los estudios geográficamente $y$, dentro de las universidades, se ha identificado en qué facultades 0 asociadas a qué estudios se asocian los estudios en Astronomía Cultural.

Por último se analizaron las competencias y conocimientos específicos de los egresados. Aptitudes, competencias y/o habilidades que se requieren para egresar de los programas en estudio. 
Asimismo para completar la imagen de la situación de la Astronomía Cultural se ha realizado una revisión de otros aspectos que conforman la disciplina, como son las revistas especializadas en el campo y las asociaciones científicas.

\section{Resultados}

Actualmente existen pocos ejemplos de grados en astronomía cultural (ver Tabla 1), comparativamente hablando con otras disciplinas. Como se comentaba anteriormente la incursión en la universidad a nivel docente es todavía tímida. De cualquier manera, se pueden dividir en tres grandes grupos, estudios de grado, estudios de posgrado y educación no formal.

Entre los estudios de grado tenemos 5 relacionados con la astronomía cultural que se dividen en 4 asignaturas optativas generales y una obligatoria en una licenciatura de otra disciplina. Entre los estudios de posgrado tenemos 4 que se dividen en una asignatura optativa en un programa de maestría de otra disciplina, una asignatura obligatoria en una maestría de otra disciplina, una maestría con temática en astronomía cultural y un programa de doctorado en Astronomía Cultural. Por último, como estudios no formales tenemos un diplomado y 2 seminarios de investigación.

Geográficamente los estudios se dividen en las dos regiones que históricamente han tenido un mayor número de estudios en Astronomía cultural, Europa y América (ver figura 1).

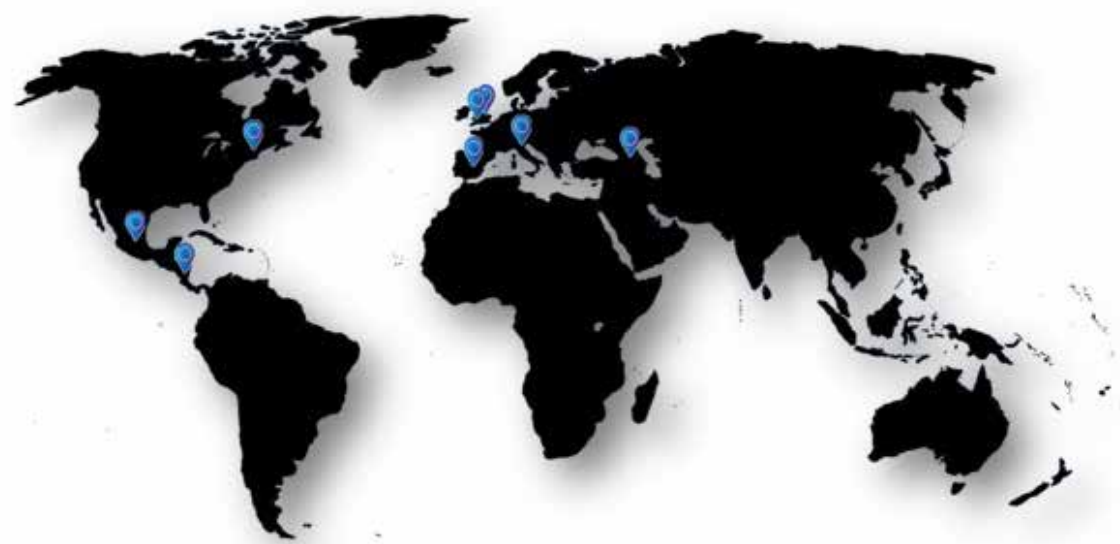

Figura 1. Lugares dónde se encuentran los estudios en Astronomía Cultural. 
En Europa los estudios con más tradición se sitúan en Inglaterra, en la facultad de Arqueología e Historia antigua de la Universidad de Leicester. Los llamados courses in Archaeoastronomy encierran una asignatura optativa general de grado que cubre aspectos introductorios de la disciplina y que viene siendo llevado a cabo por Clive Ruggles. Es un curso de 11 semanas divido en cuatro bloques temáticos: naturaleza y desarrollo de la Arqueoastronomía, Astronomía en la Gran Bretaña e Irlanda prehistóricas, Arqueoastronomía en el mundo y salidas de campo.

También optativas generales son las asignaturas del único departamento universitario de la disciplina, el Departamento de Arqueoastronomía y Astronomía Cultural en la Facultad de Ciencias Espaciales de la Universidad Nacional Autónoma de Honduras. Estás dos asignaturas son: Introducción a la Arqueoastronomía e Introducción a la Arqueoastronomía Maya, como su propio nombre indica son de carácter introductorio con una fuerte componente de conocimiento en culturas mesoamericanas. Las asignaturas -que son elegibles a nivel de grado- se imparten 3 veces en el año académico con varias secciones para cada una de las asignaturas, sobrepasan los 300 alumnos en el curso en ambos casos.

El siguiente curso, Archeoastronomia, que imparte Giulio Magli se incluye en los planes de estudio de la licenciatura de Diseño Arquitectónico del Instituto Politécnico de Milán. Tiene un fuerte componente de las culturas romana y egipcia, debido a que son las áreas en las que ha venido Magli investigando durante su carrera profesional.

Las últimas asignaturas de grado son las que Aveni imparte en la Universidad de Colgate dentro de los Estudios Nativo-Americanos. Las dos asignaturas: Astronomy in culture y Comparative cosmologies forman parte de las asignaturas optativas elegibles para los egresados del programa de estudios nativo-americanos, como es obvio, tratan aspectos de culturas indígenas americanas y no se ocupan de otros nichos culturales o geográficos.

Cambiando ahora a los estudios de posgrado, comenzaremos con la asignatura optativa Exploración geofísica y orientación topoastronómica de yacimientos arqueológicos, dentro del Máster en Geofísica de la Facultad de Ciencias Físicas de la Universidad Complutense de Madrid. Es impartida por María de Gracia Rodríguez-Caderot y María Luisa Cerdeño que dirigen, a su vez, el grupo de investigación en arqueoastronomía de la Universidad Complutense de Madrid, que con el que dirige Juan Antonio Belmonte en Canarias son los dos grupos actuales de investigación en el campo más relevantes en España. 
De nuevo en el Departamento de Arqueoastronomía y Astronomía Cultural de Honduras nos encontramos con una asignatura, Seminarios en Arqueoastronomía, dentro del programa de la Maestría Académica Regional Centroamericana en Astronomía y Astrofísica (MARCAA). Se trata de una asignatura basada en la investigación que se realiza en el propio departamento y destinada a que los alumnos conozcan las nociones básicas disciplinares así como las técnicas propias de la Astronomía Cultural.

Los dos siguientes programas de estudio son los dos únicos casos que tenemos hasta el momento que se pueden considerar como grados en Astronomía Cultural. Se trata de la Maestría en Astronomía Cultura y Astrología impartido desde 2002 (Brady y Bird, 2013; Campion y Malville, 2011), y desde 2008 solamente en modalidad virtual, dentro de la Facultad de Arqueología, Historia y Antropología de la Universidad de Gales. La maestría consta de 180 créditos ECTS, con unos módulos obligatorios de 20 créditos ECTS cada uno: Introducción a la astronomía cultural y la astrología, Métodos de investigación: Etnografía y trabajo de campo, Historia de la astrología. El curso se completa con la elección de uno de los tres itinerarios: El cosmos interior, Estrellas y piedras y Tierra y Cielo, y una asignatura de uno de los otros dos restantes junto con una disertación.

Para finalizar con la educación formal tenemos el programa de doctorado de la Escuela de Artes y Ciencias de la llia State University que incluye en su plan de estudios: Curso especial de Astronomía, Astronomía Cultural y Arqueoastronomía, Arqueología del Paisaje, Historia antigua. Según la información que muestra la dirección del doctorado se trata de un programa de 4 años gratuito.

\section{Estudios en Astronomía Cultural}

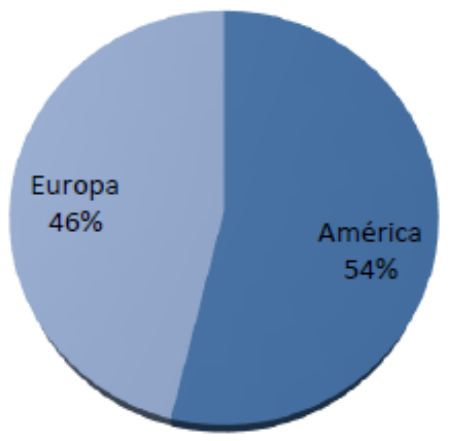

Figura 2. Distribución geográfica de los estudios en Astronomía Cultural 
La educación no formal ha sido importante en la conformación actual de la disciplina ya que ha sido una manera de introducirse en el ámbito académico en muchas universidades y centros de investigación. En este capítulo tenemos el Diplomado en Arqueoastronomía Maya, del ya comentado Departamento de Arqueoastronomía y Astronomía Cultural hondureño, y los seminarios de investigación que dirigen en la Universidad Nacional Autónoma de México, Jesús Galindo Trejo y Stanislaw Iwaniscewski dentro de la Escuela Nacional de Antropología e Historia. El otro seminario de investigación es el que lleva a cabo Clive Ruggles en Leicester como complemento a los Courses in Archaeoastronomy comentados previamente.

\section{Frecuencia}

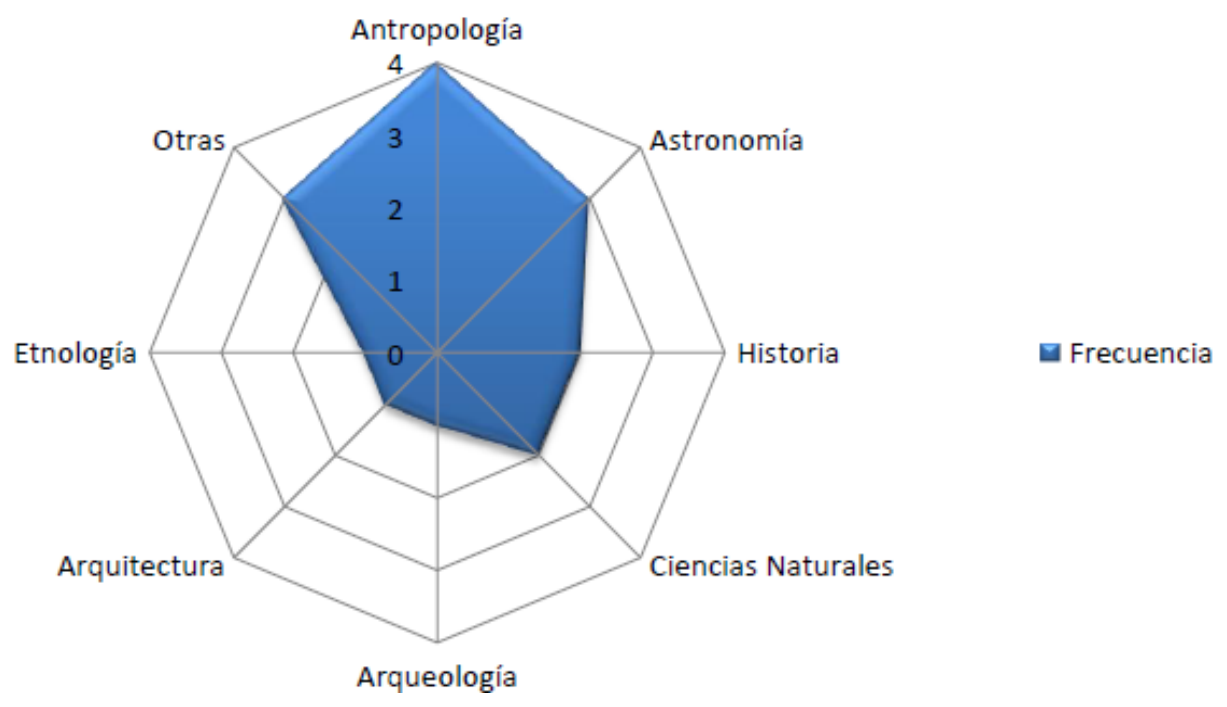

Figura 3. Frecuencia de asignaturas relacionadas con los requisitos de entrada a los estudios

Se trata de un total de 13 casos, entre estudios de grado (46.2\%), posgrado $(30.7 \%)$ y educación no formal $(23.1 \%)$. De ellas el $46.2 \%$ son optativas de algún tipo y solamente el $15.4 \%$ conforman grado académico y ninguna de ellas de licenciatura (ver figura 4). Geográficamente el $46.2 \%$ se imparten en Europa y el $53.8 \%$ en América (ver figura 2). 


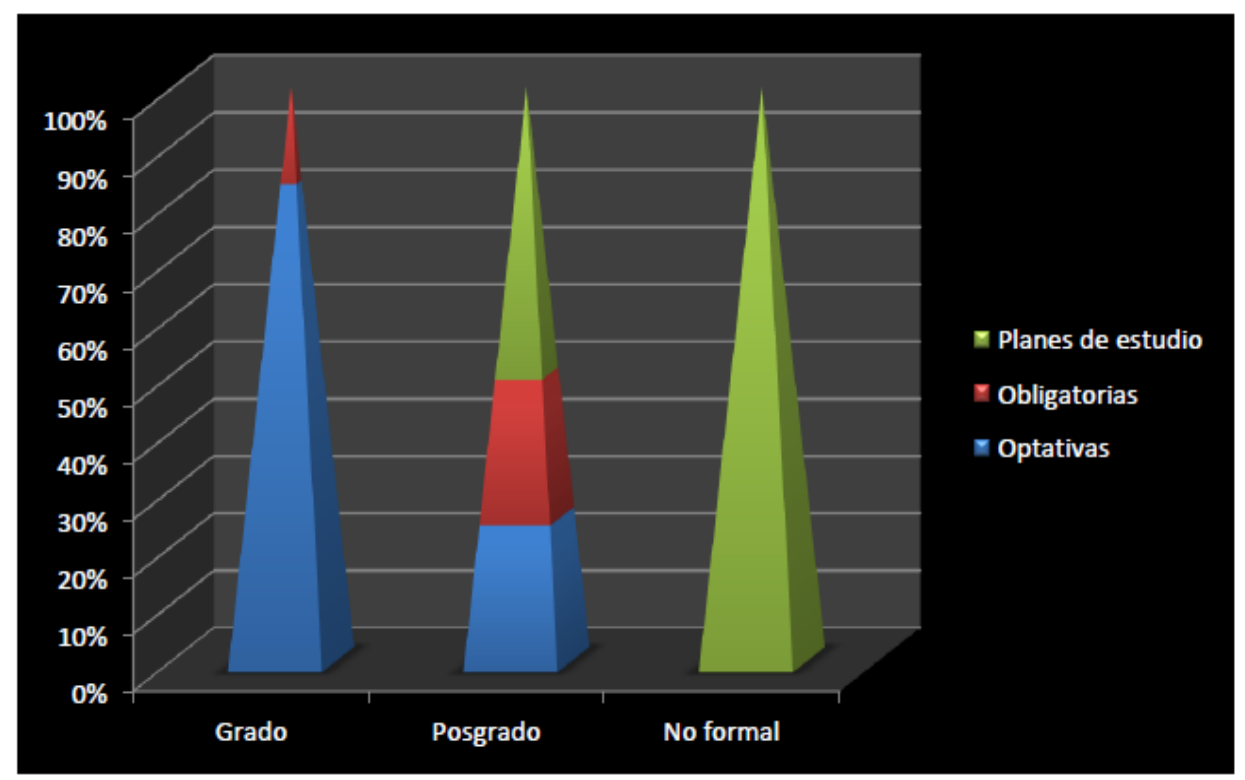

Figura 4. Reparto de estudios optativos, obligatorios y planes de estudio propios entre niveles de grado, posgrado y educación no formal

En cuanto a los requisitos de entrada a los estudios, solamente el $38.2 \%$ especifican algún requisito entre los que destacan Antropología (23.5\%), Astronomía (17.6\%), Historia (11,8\%). Tras ellos aparecen Arqueología, Física, Matemáticas, Inglés, Arquitectura, Etnología y otras Ciencias Sociales y Humanidades, todos ellos aparecen en un $5.8 \%$ de las ocasiones (ver figura 3).

Los estudios se sitúan en facultades de Arqueología, Historia o Antropología en el $33.3 \%$ de las ocasiones, el $50 \%$ en las facultad de Ciencias y el $16.7 \%$ en facultades de Arquitectura.

\section{Discusión}

Los cursos y grados en Astronomía Cultural, al igual que los trabajos científicos aunque van creciendo de forma muy sustancial en África y Oceanía, están polarizados en los continentes europeo y americano pero Europa está perdiendo la hegemonía en este campo que siempre tuvo debido a que fue en este continente dónde comenzó la disciplina. Dentro de esta polarización es América la que posee un mayor número de cursos, siendo Honduras con el $57.1 \%$ el lugar más propicio para estudiar Astronomía Cultural en cuanto a número y variedad de cursos espe- 
cíficos. De hecho, al ser el único departamento universitario del campo se abren unas posibilidades de desarrollo disciplinar a través de él que no existían hasta el momento.

Sin embargo en los estudios, como vemos, no existe una licenciatura propia en Astronomía Cultural. Es reseñable que existan estudios en todos los demás grados educativos superiores salvo a nivel de licenciatura. Sin embargo, ello responde a la propia concepción de la disciplina, que ha venido siendo una disciplina que se encuentra a caballo entre dos paradigmas científicos muy diferentes y marcados (la Astronomía y la Arqueología), que ha ocasionado ciertos problemas típicos de los trabajos interdisciplinares. Al no considerarse, hasta el momento, como una disciplina independiente se ha ido incluyendo en otros planes de estudio, como asignatura optativa o como conocimientos complementarios a disciplinas como la antropología y la arqueología, entre otras.

Si bien es cierto que podríamos considerar a la Astronomía Cultural como disciplina auxiliar de otras ciencias, no es menos cierto que es y debe considerarse una disciplina con entidad propia epistemológica y metodológicamente diferenciada. En la actualidad, la situación que podemos ver a través de los datos se corresponde de forma extraordinaria con la historia de la disciplina. Desde los inicios, han sido ciertos investigadores -Michael Hoskin, Aveni, Clive Ruggles, en el ambiente anglosajón y otros como María Cristina Pineda, Santos Vito Véliz o Ricardo Agurcia en Honduras o Juan Antonio Belmonte, Gracia Rodríguez-Caderot y María Luisa Cerdeño en España- los que a través de sus intereses y trabajo han creado en su entorno núcleos de investigación que, a su vez, germinaron en grupos de investigadores que continuaron el trabajo ampliándolo en número de estudios, países y culturas estudiadas. 


\begin{tabular}{|c|c|c|c|c|}
\hline TIPO & NOMBRE & CARÁCTER & FACULTAD & UNIVERSIDAD \\
\hline \multirow[t]{6}{*}{ GRADO } & $\begin{array}{l}\text { Courses in } \\
\text { Archaeoastronomy }\end{array}$ & $\begin{array}{l}\text { Optativa } \\
\text { General }\end{array}$ & $\begin{array}{l}\text { School of Archaeology } \\
\text { \& Ancient History }\end{array}$ & University of Leicester \\
\hline & $\begin{array}{l}\text { Introducción a la } \\
\text { Arqueoastronomía }\end{array}$ & $\begin{array}{l}\text { Optativa } \\
\text { General }\end{array}$ & $\begin{array}{l}\text { Facultad de Ciencias } \\
\text { Espaciales }\end{array}$ & $\begin{array}{l}\text { Universidad Nacional } \\
\text { Autónoma de } \\
\text { Honduras }\end{array}$ \\
\hline & $\begin{array}{l}\text { Introducción a la } \\
\text { Arqueoastronomía } \\
\text { Maya }\end{array}$ & $\begin{array}{l}\text { Optativa } \\
\text { General }\end{array}$ & $\begin{array}{l}\text { Facultad de Ciencias } \\
\text { Espaciales }\end{array}$ & $\begin{array}{l}\text { Universidad Nacional } \\
\text { Autónoma de } \\
\text { Honduras }\end{array}$ \\
\hline & $\begin{array}{l}\text { Astronomy in } \\
\text { Culture }\end{array}$ & $\begin{array}{l}\text { Optativa } \\
\text { (Native } \\
\text { American } \\
\text { Studies) }\end{array}$ & & Colgate University \\
\hline & $\begin{array}{l}\text { Comparative } \\
\text { Cosmologies }\end{array}$ & $\begin{array}{l}\text { Optativa } \\
\text { (Native } \\
\text { American } \\
\text { Studies) }\end{array}$ & & Colgate University \\
\hline & Archeoastronomia & $\begin{array}{l}\text { Obligatoria } \\
\text { (Architectural } \\
\text { Design) }\end{array}$ & $\begin{array}{l}\text { Scuola di Architettura } \\
\text { Civile }\end{array}$ & Politecnico de Milano \\
\hline \multirow[t]{4}{*}{ POSGRADO } & $\begin{array}{l}\text { Exploración } \\
\text { geofísica y } \\
\text { orientación } \\
\text { topoastronómica } \\
\text { de yacimientos } \\
\text { arqueológicos }\end{array}$ & $\begin{array}{l}\text { Optativa } \\
\text { (Máster en } \\
\text { Geofísica) }\end{array}$ & $\begin{array}{l}\text { Facultad de Ciencias } \\
\text { Físicas }\end{array}$ & $\begin{array}{l}\text { Universidad } \\
\text { Complutense de } \\
\text { Madrid }\end{array}$ \\
\hline & $\begin{array}{l}\text { Seminarios en } \\
\text { Arqueoastronomía }\end{array}$ & $\begin{array}{l}\text { Obligatoria } \\
\text { (MARCAA) }\end{array}$ & $\begin{array}{l}\text { Facultad de Ciencias } \\
\text { Espaciales }\end{array}$ & $\begin{array}{l}\text { Universidad Nacional } \\
\text { Autónoma de } \\
\text { Honduras }\end{array}$ \\
\hline & $\begin{array}{l}\text { MA Cultural } \\
\text { Astronomy and } \\
\text { Astrology }\end{array}$ & & $\begin{array}{l}\text { Sophia center for the } \\
\text { study of cosmology in } \\
\text { culture }\end{array}$ & University of Wales \\
\hline & $\begin{array}{l}\text { Ph.D program in } \\
\text { cultural astronomy } \\
\text { and } \\
\text { archaeoastronomy }\end{array}$ & & $\begin{array}{l}\text { School of Arts \& } \\
\text { Sciences }\end{array}$ & Ilia State University \\
\hline \multirow[t]{3}{*}{$\begin{array}{l}\text { NO } \\
\text { FORMAL }\end{array}$} & $\begin{array}{l}\text { Diplomado en } \\
\text { Arqueoastronomía } \\
\text { Maya }\end{array}$ & & $\begin{array}{l}\text { Facultad de Ciencias } \\
\text { Espaciales }\end{array}$ & $\begin{array}{l}\text { Universidad Nacional } \\
\text { Autónoma de } \\
\text { Honduras }\end{array}$ \\
\hline & $\begin{array}{l}\text { Seminarios en } \\
\text { Arqueoastronomía }\end{array}$ & & $\begin{array}{l}\text { Escuela Nacional de } \\
\text { Antropología e } \\
\text { Historia }\end{array}$ & $\begin{array}{l}\text { Universidad Nacional } \\
\text { Autónoma de México }\end{array}$ \\
\hline & $\begin{array}{l}\text { School Research } \\
\text { Seminar Series }\end{array}$ & & $\begin{array}{l}\text { School of Archaeology } \\
\text { \& Ancient History }\end{array}$ & University of Leicester \\
\hline
\end{tabular}

Tabla 1. Estudios en Astronomía Cultural. Para más información ver Anexo I. 
La centralización en investigadores individuales, aunque lógica en el momento que se encuentra la disciplina, ha tenido como consecuencia que los estudios en arqueoastronomía sean sesgados y faltos de estandarización. Por ejemplo, la asignatura del Instituto Politécnico de Milán se enfoca especialmente en la cultura egipcia, ya que es ésta la especialidad del profesor Magli que la imparte. Faltan por lo tanto, un verdadero plan de estudios que sea integrador y permita obtener al egresado una visión general y completa de la disciplina, consiguiendo así crear un verdadero perfil profesional de astrónomo cultural.

A pesar de que existen tres grandes asociaciones profesionales: SEAC (Sociedad Europea de Astronomía Cultural), SIAC (Sociedad Interamericana de Astronomía Cultural), ISAAC (International Society for Archaeoastronomy and Astronomy in Culture), no ha surgido entre ellas un grupo de profesionales que acometa esta estandarización disciplinar y la verdadera inclusión del campo en la comunidad universitaria.

Otro ejemplo de la necesidad de estandarizar y estabilizar los estudios dentro de la universidad son las revistas científicas del campo. Inicialmente los trabajos se fueron publicando en el suplemento -ya extinto- de la Journal for the History of Astronomy. En la actualidad, existen otras revistas como Archaeoastronomy: the journal of astronomy in culture, sin embargo la mayor parte de los artículos deben publicarse en revistas no específicas del campo haciendo difícil su seguimiento y la discusión entre profesionales mediante sus páginas. Como vemos, la subsidiariedad disciplinar actual de la Astronomía Cultural se refleja en los aspectos de investigación como docencia.

Si nos detenemos en los datos que arroja el presente estudio, vemos -la ya comentada- preponderancia de los estudios optativos o incluidos en otras carreras, solamente el $15.4 \%$ de los estudios conforman grado propio en Astronomía Cultural. Por otro lado, se puede apreciar el carácter interdisciplinario tan defendido por muchos autores. En especial, podemos definir como campos afines la Antropología, Arqueología, Astronomía e Historia en primera instancia, seguidos por una serie de campos de las Ciencias Sociales, Naturales y Humanidades. En cuanto a los lugares donde se imparten estos estudios es casi de forma exclusiva en facultades de ciencias y facultades de Arqueología e Historia, de forma coherente con las disciplinas afines a la Astronomía Cultural.

Debido al carácter multidisciplinar de la Astronomía Cultural el diseño curricular de un plan de estudios de una licenciatura presenta mayores dificultades que el diseño de licenciaturas de campos clásicos. Aún con estas dificultades es 
posible proponer un esbozo de plan de estudios modular basado en las ciencias y disciplinas afines de la Astronomía Cultural, junto con asignaturas que enlacen y relacionen conceptos transdisciplinares. También son de relevancia asignaturas específicas de metodología de trabajo de campo y análisis posterior de datos. Finalmente son necesarias aquellas asignaturas que permitan un manejo de las nuevas tecnologías con la finalidad de un manejo correcto de datos como el desarrollo y manejo de software específico.

Si queremos, como se propone en estas líneas, la creación de unos estudios en grado de licenciatura no debemos olvidar el perfil del egresado que demanda el campo, así como su nicho profesional. Aunque mayoritariamente, en estos momentos, la principal salida profesional se encuentra en la academia a través de la investigación, ya hemos visto por el número creciente de cursos y estudios que la docencia demandará cada vez más profesionales del campo. Qué duda cabe que los profesionales egresados de la Licenciatura en Astronomía Cultural serán profesionales en un campo muy específico, lo cual podría pensarse que les reducirá las posibilidades de encontrar un empleo. Sin embargo, tenemos ejemplos que avalan las posibilidades de colocarse en el mundo laboral. Ejemplos de ello son los estudios mesoamericanos, estudios indígenas de diversos tipos, arqueometrías como la arqueometalurgia, arqueobotánica, etcétera. Estos son ejemplos de especialistas que son incluidos en grandes proyectos científicos de muy diversa índole.

Además de estos campos, más o menos tradicionales, últimamente se están abriendo grandes oportunidades laborales en los campos del turismo cultural arqueológico (Alonso López, 2008) y el patrimonio. El patrimonio cultural considera los monumentos: obras arquitectónicas, elementos o estructuras de carácter arqueológico, inscripciones, cavernas y grupos de elementos; los conjuntos: grupos de construcciones, aisladas o reunidas, cuya arquitectura, unidad e integración en el paisaje les dé un valor universal; y los lugares: obras del hombre u obras conjuntas del hombre y la naturaleza así como las zonas, incluidos los lugares arqueológicos; todos ellos que tengan un valor excepcional desde el punto de vista de la historia, el arte, lo estético, etnológico, antropológico o de la ciencia. Una nueva categoría patrimonial del máximo interés para la Astronomía Cultural es el Patrimonio Astronómico. En este concepto trabajan la UNESCO, el International Council on Munuments and Sites (ICOMOS) y el Grupo de Astronomía y Patrimonio de la Humanidad de la Unión Astronómica Internacional. El Patrimonio Astronómico es la evidencia relativa a la práctica de la Astronomía y los usos y representaciones sociales de la misma. Comprende cuatro categorías: monumentos y sitios, tangible y fijo; instrumentos y artefactos, tangible móvil; conocimientos e ideas, intangible 
cultural; y paisaje y cielos, natural (UNESCO and World Heritage Center, 2014). Como es obvio, esto abre unas posibilidades laborales para los futuros astrónomos culturales en los campos del turismo cultural y las ciencias del patrimonio que hasta la actualidad no existían.

\section{Conclusión}

La Astronomía Cultural se encuentra en un momento decisivo para su definición futura como campo científico. Es el momento de tener una disciplina estandarizada, libre de los problemas que, como disciplina emergente e interdisciplinar, ha padecido.

La creación de una Licenciatura en Astronomía Cultural permitirá que los planes de estudio se estandaricen, permitiendo un perfil único de profesional del campo, cerrando con ello la brecha que existe entre estudios de grado y posgrado. Como consecuencia aparecerá una disciplina bien diferenciada con carácter multi e interdisciplinar. Se evitará la dependencia directa de la formación del alumnado por los intereses científicos del profesorado. Al crearse una comunidad homogénea de profesionales parece inmediato que las redes profesionales y revistas especializadas se verán ampliadas y desarrolladas por una mayor cantidad de profesionales siempre compartiendo un mismo marco conceptual.

Este trabajo ha permitido definir las llamadas disciplinas afines de la Astronomía Cultural y que nos permitirán conformar el currículo de la futura licenciatura dentro de los parámetros teóricos disciplinares. Que exista unos estudios propios en el grado de licenciatura que ayuden a conformar de forma definitiva y estable la disciplina no implica abandonar el carácter interdisciplinar de la Astronomía Cultural, si no que debemos reforzar y afianzar estos aspectos a través de la estandarización de los estudios, siempre abiertos a una ciencia dinámica. Creo que la creación de este tipo de estudios solamente puede redundar en una mejora de la Astronomía Cultural en todos los aspectos, produciendo trabajos de una gran calidad científica que permita el reconocimiento del resto de campos científicos con los que compartimos objetos de estudio.

\section{Bibliografía}

- Alonso López, O. (2008) Turismo Arqueoastronómico: Diseño de una Ruta Turística arqueoastronómica en la Zona Arqueológica de Teotihuacan como un Servicio Turístico Innovador. Tesis de Maestría. 
- Baity, Elizabeth (1973) Archaeoastronomy and Ethnoastronomy So Far, Current Anthroplogy, Vol. 14, № 4.

- Brady, B. y Bird, A. (2013) "A Niche Degree: a Case Study of an MA (in Cultural Astronomy and Astrology". Diskus 14: 55-69

- Cerdeño, M. L.; Rodríguez-Caderot, G.; R. Moya, P.; Ibarra, A. y Herrero, S. (2006). "Los estudios de arqueoastronomía en España: Estado de la cuestión". Trabajos de Prehistoria, 63, 2, pp. 13-34.

- Campion, N., Malville, M. (2011). Masters-level education in archaeoastronomy at the University of Wales Trinity Saint David "Oxford IX" International Symposium on Archaeoastronomy. International Astronomical Union Proceedings IAU Symposium No. 278, 2011. Clive L. N. Ruggles, ed. doi:10.1017/ S1743921311012804

- Iwaniszewski, S. (1991). "Astronomy as a Cultural System". Interdisciplinarni izsledvaniya, 18, pp. 282-288.

- _ _ (1994). "De la astroarqueología a la astronomía cultural". Trabajos de Prehistoria, 51 (2), pp. 21-39.

- Pineda de Carías, M. C., Vito Veliz y Ricardo Agurcia Fasquelle (2002a). "Del grande y complejo plan de 18 Conejo para la Plaza del Sol del Parque Arqueológico de Copán Ruinas". Revista del Instituto Hondureño de Antropología e Historia. Edición Conmemorativa 50 Aniversario: 1952-2002:126-134. Instituto Hondureño de Antropología e Historia. Tegucigalpa, Honduras.

- - (2002b). "Acerca de las observaciones del Sol realizadas en la Gran Plaza del Parque Arqueológico de Copan Ruinas, Honduras". Revista Yaxkin, Volumen XXI:15-44. Instituto Hondureño de Antropología e Historia. Tegucigalpa, Honduras.

- _ - (2009). "Estela D: Reloj Solar de la Plaza del Sol del Parque Arqueológico de Copán Ruinas, Honduras". Revista Yaxkin. Instituto Hondureño de Antropología e Historia. Año 34, Vol. XXV, No.2: 111-138.

- UNESCO and World Heritage Center. (2 de Julio de 2014). Portal to the Heritage of Astronomy. Recuperado el 2 de julio de 2014, de Portal to the Heritage of Astronomy: http://www2.astronomicalheritage.net/index.php/what-is-astronomical-heritage 
- Williamson, R.A. (1981). "Archaeoastronomy in the Americas". Ballena Press y Center for Archaeoastronomy. Los Altos.

\section{ANEXO I}

\section{ESTUDIOS EN ASTRONOMÍA CULTURAL}

- UNIVERSIDAD NACIONAL AUTÓNOMA DE HONDURAS

http://faces.unah.edu.hn/arqueo

http://faces.unah.edu.hn/astro/marcaa/

- UNIVERSIDAD COMPLUTENSE DE MADRID

http://pendientedemigracion.ucm.es/info/Geofis/webmaster/yacimientos_arqueolo. $\mathrm{htm}$

- UNIVERSIDAD DE LEICESTER

http://www.uwtsd.ac.uk/ma-cultural-astronomy-astrology/

http://www2.le.ac.uk/departments/archaeology/research/seminars

- UNIVERSITY OF WALES

http://www.uwtsd.ac.uk/ma-cultural-astronomy-astrology/

- ILIANA STATE UNIVERSITY

http://www.iliauni.edu.ge/index.php?sec_id=932\&lang_id=ENG\#3

- INSTITUTO POLITECNICO DE MILANO

https://www4.ceda.polimi.it/manifesti/manifesti/controller/extra/RegolamentoPublic.do?jaf_currentWFID=main\&EVN_DEFAULT=evento\&k_corso_ $l a=1118 \& a a=2014 \& l a n g=E N$

- COLGATE UNIVERSITY

http://www.colgate.edu/academics/course-catalogue/native-american-studies 